\title{
Are UK cancer cure rates worse than in most other European countries?
}

The review from William Hamilton ${ }^{1}$ in the current issue of the BJGP is a valuable and careful analysis of where delays might occur in the diagnosis of cancer. The review examines the implications for appropriate management when patients first present with symptoms that could be due to many causes, including cancer.

Early diagnosis of cancer is essential and delay may reduce the chance of cure. Delay in diagnosis is not a feature exclusive to the UK healthcare system. It is often very difficult to determine when the first symptoms of a cancer occur, so that comparing treatment delays between one UK practice and another, let alone between countries, is a difficult thing to do.

There is no reason to suppose that UK doctors are worse trained or more careless than those in other countries. Of course, if there are identified, verifiable, institutional delays, these must be rectified and it is encouraging to see the steps that are now being taken to do this. ${ }^{2}$

To illustrate the importance of timely diagnosis, Hamilton refers to studies that state that UK cure rates are below those in many European countries. This widely quoted assertion comes from the findings in the EUROCARE studies. ${ }^{3,4}$ However the EUROCARE data are susceptible to several potential biases that mean that we must interpret the studies with caution. The need for rapid diagnosis of cancer stands on its own and does not rest on the basis of the EUROCARE data.

The EUROCARE-4 data are the latest in a programme of studies based on data collected from participating European cancer registries. The aims are to determine whether there are differences in cure rates for cancers in European countries, whether any such differences and cure rates are changing with time, and to relate any observed differences to national expenditure on health care. The ultimate aim would be to identify patterns of delivery of care that either facilitate or impede cancer cure. If this could be done with possible biases and confounding factors eliminated, this would indeed be a worthwhile enterprise.

The latest data were reported in an abbreviated form in $2007^{3}$ and in much more detail in a recent supplement. ${ }^{4}$ They refer to patients diagnosed with cancer in the period 1995-1999 and followed up in 2003. As will be seen later in the editorial, this time period is of particular importance in considering the data. There is an inherent likelihood of bias when survival rates are compared by observational data derived from general populations. Mortality data refer to the death rate from a disease in a population. Survival rates, in contrast, refer to the proportion of persons who, having contracted the disease, survive over a given time period since diagnosis. Data are presented as relative survival when compared with survival with persons of the same age and sex in the population. In cancer a 5-year survival is a commonly used substitute for a cure rate, even though with some cancers notably breast cancer - death continues to occur long after 5 years.

To give an accurate estimate, we need to know the total number of persons diagnosed with the disease over a given period and how many of them have not died of the disease at 5 years. This poses great problems in data acquisition and verification, and the results are susceptible to confounding and biases. In comparing survival rates between countries we need as near as possible to $100 \%$ of patients to have been registered, preferably soon after diagnosis. If not all cancer cases are registered, then the question arises as to the persons left out. How do we know who they are? There might be a systematic bias; for example, on the grounds of occupation, social class, and/or ethnicity. Poorer members of the population, or those of a minority ethnic background, might have been less likely to have the disease registered. Furthermore, cancer survival rates may differ in one part of a country from another.

Comorbidities (vascular disease, chronic respiratory disease, and diabetes) are also unlikely to be equal in all parts of a country, or between countries. It is likely that cancer treatment decisions in areas of high comorbidity will frequently be justifiably different from those taken elsewhere. It is clear that if registries are not representative of the entire population of a country, national survival rates are very likely to be estimated incorrectly.

In the EUROCARE studies there is a remarkable failure of many European countries to register cancer. Germany is represented by the Saarland register which includes only $1 \%$ of the national population. Germany submitted to the study, from a population of 70 million, less than half the number of cases than Wales, which has a population of 2 million. Of their total national populations, the French registries contributed $17 \%$ (elsewhere quoted as $10 \%$ ) Spain $16 \%$, and Italy $28 \%$. In contrast, nine countries, including the UK, registered more than $80 \%$. Most of these latter countries are Nordic where, in some cases, registration is required by law. Apart from the UK, these countries have small populations.

The result was that the UK contribution to the entire EUROCARE study accounted, astonishingly, for more than one-third of all European cancer from only about $12 \%$ of the European population. The fact that the studies include millions of patients will not eliminate bias. On the contrary, it may give unjustified authority to debatable or erroneous conclusions. The EUROCARE-4 reports do not discuss this important issue in any detail. They refer to some in-depth 'high resolution' studies on small, registry-derived, samples $^{5,6}$ to determine the reasons for differences in outcome, but these explorations, which show predictable differences in outcome according to stage and variability in practice, do not address the question of representativeness and bias.

The likelihood of non-representativeness is illustrated within the detailed data where there are some figures that give the notional cure rates for cancers by individual registries. For France, we see that the lowest registryrecorded survival for gastric cancer is $16.2 \%$ and the highest registry figure is $36 \%$. But if, in France, only $17 \%$ of patients are registered, then what is the national survival 
rate for gastric cancer? The national average is given as $26 \%$ but we clearly cannot be confident that figure is truly representative of the national situation. These findings are unsurprising. In UK studies, Coleman and colleagues showed a $9 \%$ difference in survival between deprived and affluent regions in England. ${ }^{7}$ Adding to these difficulties is variation in aspects of cancer registration between countries. The technical issues concern the use of data from death certificates and the time dependency of the completeness of registration. ${ }^{8}$

It would therefore be most unwise to trust comparisons with France, Germany, Italy, and Spain and the countries of Eastern Europe. Can we rely on comparisons with countries where there is full registration? These were Austria, Denmark, Finland, Iceland, Ireland, Norway, Slovenia, Sweden, and the UK. The EUROCARE registry data suggest that, for a minority of cancers, the UK cure rate is below that achieved in some of these countries. But even in this comparison there are potential difficulties, especially for diseases where programmes of screening and early detection were being introduced or where outcome is related to ethnicity.

Lead-time bias arises from early diagnosis made without affecting the proportion of cases who are ultimately cured. This is a particular problem with prostate cancer, where the introduction of prostate specific antigen tests may account for an overdiagnosis rate (the proportion of cases that would never have been clinically apparent) of $30 \% .{ }^{9}$ Breast cancer screening may identify cases that will never become symptomatic in the life-time of the patient. The proportion of overdiagnosis compared with an unscreened population is about $10 \%$ in some studies. ${ }^{10}$ Therefore, in interpreting the data on prostate, colorectal, and breast cancer survival rates, it is essential to know how many of these cases have been diagnosed by these methods. These data are not known for EUROCARE-4. Yet the period in question (1995-1999) was a period of rapid and variable expansion of the use of these methods outside systems of national evaluation or accountability. The importance of this bias is shown by a subsequent critical analysis of the data. ${ }^{11}$ The combined prostate cancer incidence for Denmark and UK was 35.3 (per 100 000) and mortality was
17.7. For the eight other countries the incidence was much higher at 59.3 but the mortality was the same at 17.3. These discrepancies will have a great effect on calculated survival rates, even though mortality has not changed.

Prostate cancer is also a disease that is especially severe in men of African-Caribbean origin. Ethnicity is not recorded in registries contributing to the EUROCARE studies but, for this tumour, comparisons of cure rates in countries such as the UK (which has large and varied immigrant populations) with Finland, Sweden, or Austria are likely to be distorted.

Of the 45 cancer types analysed, there were some where there was a reported difference in survival for the UK, compared with three or more of these Nordic countries, where the confidence intervals for the 5-year survival estimate did not overlap. These were: stomach, colon and rectum, breast, cervix, ovary, prostate, bladder, and lung cancers. But screen detection was being introduced for breast and prostate cancers, and to a lesser extent colorectal cancer during this period. These differences therefore apply to five cancer sites where unquantifiable use of screening would not be implicated in the interpretation of the results. The differences with the UK vary between countries and cancers, and are usually between $4 \%$ and $9 \%$. For all cancers combined, they range from $5 \%$ to $12 \%$. These are often relatively small differences, although they would be important if they were true. But we do not know with confidence that they are.

These formidable difficulties in methodology and interpretation of national survival data derived from registry data lead some epidemiologists to the view that mortality data are a more reliable guide to outcome. Doll and Boreham ${ }^{12}$ make this point by publishing European mortality data as a response to the findings in EUROCARE-3. These showed that with the exception of three tumour sites the UK mortality was equal to, or better than, many European countries. Cancer registry survival data are most convincing when there is $100 \%$ registration and the same registries and methods are used to explore changes in survival in regions and over time all within one country.

Publication of the EUROCARE-4 data has led to a widely repeated and unchallenged acceptance that cancer care in the UK is sub-standard. This is largely the fault of the UK media, rather than the EUROCARE investigators. This has been enormously damaging for patients and for the staff of our cancer units. We, of course, must do better with early diagnosis and swift access to best management. But so must every country. We should resolve to do this as an objective that is desirable for many reasons, but not because we are working in a system of proven inadequacy.

\section{Robert Souhami,}

Robert Souhami, Emeritus Professor of Medicine, University College London.

\section{Provenance}

Commissioned; not peer reviewed.

\section{REFERENCES}

1. Hamilton W. Cancer diagnosis in primary care. $\mathrm{Br} J \mathrm{Gen}$ Pract 2010; 60(571): 121-128.

2. Richards MA, Hiom S, editors. Diagnosing cancer earlier: evidence for a National Awareness and Early Diagnosis Initiative. Br J Cancer 2009; 101 (Suppl 2): S1-S129.

3. Berrino F, De Angelis R, Sant M, et al. Survival for eight major cancers and all cancers combined for European adults diagnosed in 1995-99: results of the EUROCARE-4 study. Lancet Oncol 2007; 8(9): 773-783.

4. Sant M, Allemani C, Santaquilani M, et al. EUROCARE-4. Survival of cancer patients diagnosed in 1995-1999. Results and commentary. Eur J Cancer 2009; 45(6): 931-991.

5. Gatta G, Capocaccia R, Sant M, et al. Understanding variations in survival for colorectal cancer in Europe: a EUROCARE high resolution study. Gut 2000; 47(4): 533-538.

6. Sant M, Allemani C, Capocaccia R, et al. Stage at diagnosis is a key explanation of differences in breast cancer survival across Europe. Int J Cancer 2003; 106(3): 416-422.

7. Coleman MP, Rachet B, Woods LM, et al. Trends in socioeconomic inequalities in cancer survival in England and Wales up to 2001. Br J Cancer 2004; 90(7): 1367-1373.

8. Bullard J, Coleman MP, Robinson D, et al. Completeness of cancer registration: a new method for routine use. $\mathrm{Br} J$ Cancer 2000; 82(5): 1111-1116.

9. Etzioni R, Penson DF, Legler JM, et al. Overdiagnosis due to prostate-specific antigen screening: lessons from US prostate cancer incidence trends. J Natl Cancer Inst 2002; 94(13): 981-990.

10. Moss S. Overdiagnosis and overtreatment of breast cancer: overdiagnosis in randomised controlled trials of breast cancer screening. Breast Cancer Res 2005; 7(5): 230-234.

11. Autier P, Boniol M, Héry C, et al. Cancer survival statistics should be viewed with caution. Lancet Oncology 2007; 8(12): 1050-1052.

12. Doll $\mathrm{R}$, Boreham J. Recent trends in cancer mortality in the UK. Brit J Cancer 2005; 92(7): 1329-1335.

DOI: 10.3399/bjgp10X483102

\section{ADDRESS FOR CORRESPONDENCE}

Robert Souhami

University College London, 135 Rosebery

Road, London N10 2LD.

E-mail: robert.souhami@btinternet.com 